\title{
Tanshinone IIA Promotes M2 Microglia by ER $\beta /$ IL- 10 Pathway and Attenuates Neuronal Loss in Mouse TBI Model
}

This article was published in the following Dove Press journal: Neuropsychiatric Disease and Treatment

\author{
Mingrui Chen' \\ Qiulin Chen' \\ Tao Tao ${ }^{2}$ \\ 'Department of Neurosurgery, \\ Chongqing Red Cross Hospital (People's \\ Hospital of Jiangbei District), Jiangbei, \\ Chongqing 400020, People's Republic of \\ China; ${ }^{2}$ Department of Rehabilitation \\ Medicine, Guizhou Provincial People's \\ Hospital, Guiyang, Guizhou 550002 , \\ People's Republic of China
}

Purpose: Traumatic brain injury (TBI) is a major cause of morbidity and mortality worldwide. Increasing evidence indicates that activated microglia play an important role in the inflammatory response in TBI. Inhibiting M1 and stimulating M2 activated microglia have protective effects in several animal models of central nervous system (CNS) disorders. In the present study, we investigated whether tanshinone IIA (TNA) protects neurons by shifting microglia polarization in a mouse TBI model and further investigated the mechanism in vitro.

Materials and Methods: Forty C57BL/6 mice were used to investigate the effect of TNA on microglia polarization in TBI. BV-2 cells were used to examine the mechanism of TNA in regulating microglia polarization.

Results: Normal saline (NS), TNA and the combination of TNA with ICI 182,780 (ICI, an estrogen receptor antagonist) were used to treat the TBI mice. After TBI, mice from each group demonstrated functional improvement. The improvement rate in mice treated with TNA was faster than other groups. ICI partially reversed the benefits from TNA treatment. TNA treatment significantly reduced TBI-induced neuronal loss. The number of microglia after TBI was not significantly changed by TNA treatment. However, TNA treatment significantly decreased M1 macrophage markers (iNOS, TNF $\alpha$ and IL-1 $\beta$ ) and increased M2 macrophage markers (CD206, arginase 1 and Ym1). This effect was partially abolished by ICI. TNA treatment downregulated M1 macrophage markers and upregulated M2 macrophage markers in BV-2 cells under LPS stimulation. IL-10 was significantly increased by TNA treatment without a significantly change of IL-4 and IL-13 expression. IL-10 knockdown completely abolished the effect of TNA on microglial M2 polarization.

Conclusion: Taken together, our data demonstrated that TNA attenuates neuronal loss in mouse TBI model and promotes M2 microglia by ER $\beta /$ IL-10 pathway. Thus, TNA could be a potential drug for TBI and/or the disorders that caused by microglial over-activation in CNS. Keywords: tanshinone IIA; TNA, traumatic brain injury; TBI, microglia, interleukins, macrophage polarization

\section{Introduction}

Traumatic brain injury (TBI) defines as a disruption of normal brain function caused by an external physical force and it is a major cause of disability and mortality worldwide. ${ }^{1,2}$ In China, the population-based mortality of TBI is estimated to be about $13 / 100,000$ people. $^{3}$ In the US and Europe the number of patients with TBI-related complications is about 13 million. ${ }^{4}$ Although TBI subtypes differ in their pathology, consequences a contusion core due to cell death and 
inflammatory processes are the major hallmarks of TBI at the lesion zone. ${ }^{5}$ Till now few clinical treatments for TBI have been shown to be effective or promoted functional recovery after TBI. ${ }^{6,7}$ Therefore, there is a need for novel pharmacotherapies that are able to promote neural function in TBI patients.

In TBI the inflammatory response is a key factor in the cascade of secondary injury. TBI-induced inflammatory response is characterized by activation of resident immune cells and recruitment of peripheral leukocytes into brain parenchyma. ${ }^{8,9}$ Microglia are the innate immune cells of the CNS and are the major mediators of neuroinflammation. ${ }^{10}$ Microglia respond to pro-inflammatory molecules, such as bacterial lipopolysaccharide (LPS) or interferon- $\gamma$ (IFN $\gamma$ ), to adopt a "classical" M1-like phenotype (pro-inflammatory microglia), which produces high levels of pro-inflammatory cytokines (IL-1 $\beta$, tumor necrosis factor- $\alpha(\mathrm{TNF} \alpha)$, chemokines and reactive oxygen species. ${ }^{11,12} \mathrm{M} 1$ microglia markers include iNOS, IL-1 $\beta, \mathrm{TNF} \alpha, \mathrm{CD} 86$, and MHC II. A dysregulated or excessive M1-like activation can induce neurotoxicity due to release of pro-inflammatory factors and neurotoxic mediators that set off vicious cycles of microglialmediated neurodegeneration. ${ }^{13}$ Microglia significantly polarize to the M1 phenotype and express a large number of proinflammatory factors after TBI. ${ }^{14}$ Microglia also respond to TH2 cytokines such as IL-4 and IL-13 to induce M2 activation that is associated with tissue repair, immunity against parasites, and growth stimulation. ${ }^{11,12}$ M2-polarized cells upregulate several phenotypic markers such as arginase 1, CD206, Ym1, Fizz1, and increase production of scavenger receptors for phagocytosis. ${ }^{11,12}$ Strategies shifting the M2/M1 ratio already showed promising results in variety of brain injury including $\mathrm{TBI}^{15}$ Promoting M2-like microglia is antiinflammatory and can promote regeneration. ${ }^{16-18}$ Transplanting M2-polarized cells to counteract the M2 to M1 transition after TBI has been shown to reduce neurodegeneration and promote long-term functional recovery. ${ }^{19,20}$

Tanshinone IIA (TNA) is an important lipophilic diterpene extracted from Salvia miltiorrhiza BUNGE. Since TNA has anti-inflammatory and anti-apoptotic effects it is currently used to treat patients suffering from myocardial infarction (MI), stroke, angina pectoris, diabetes, and other conditions in China and other neighboring countries. $^{21-23}$ TNA could exert an anti-inflammatory effect on LPS-induced macrophages by decreasing TLR4MyD88-NF- $\mathrm{B}$ signaling pathway and by regulating a series of cytokine production and miRNA expression. $^{24,25}$ In a spinal cord injury (SCI) rat model
TNA inhibited the expression of pro-inflammatory factors and related pathways, ameliorated apoptosis, and reversed the imbalance of the redox state. ${ }^{26}$ A previous study also demonstrated the anti-inflammatory and anti-apoptotic roles of TNA by up-regulating miR-124 and then inactivating JNK and p38 MAPK pathways. ${ }^{27}$

It has been reported that estrogen receptor $\beta(\mathrm{ER} \beta)$ but not ER $\alpha$ is expressed in microglia in the CNS. ${ }^{28} \mathrm{ER} \beta$ agonist had an anti-inflammatory effect by inhibiting M1 microglia in experimental autoimmune encephalomyelitis mice. $^{28,29}$ Stimulating ER $\beta$ in macrophages in crown-like structure (CLS) within adipose tissue decreased the number of CLS by suppressing M1 macrophages. ${ }^{30}$ Furthermore, TNA has been shown to protect myocardial ischemia reperfusion injury-induced ventricular remodeling by upregulating ER $\beta$ via PI3K/Akt signaling. ${ }^{31}$ However, no study reports whether TNA is able to shift microglia polarization through ER $\beta$ signaling.

In the present study, by using TBI mouse model and BV-2 cells, we found that TNA attenuated neuronal loss in mouse TBI model and promoted microglial M2 polarization. Further studies demonstrated that the effect of TNA on microglial M2 polarization is via ER $\beta / \mathrm{IL}-10$ signaling.

\section{Materials and Methods}

In the present study, we have followed all the guidelines stated in Guide for the Care and Use of Laboratory Animals, prepared by the Committee on Care and Use of Laboratory Animals of the Institute of Laboratory Animal Resources Commission on Life Sciences, National Research Council, China (1996). The animal studies were approved by the local Animal Experimentation Ethics Committee (Guizhou Provincial People's Hospital, Guizhou, China) for animal experimentation. Efforts were made to minimize the number of mice used and their suffering.

\section{Traumatic Brain Injury Mouse Model}

Male mice, weighing 22-27 g (8-10 weeks old), C57BL/6 were used in this study. The temperature in the room was $25{ }^{\circ} \mathrm{C}$ and the room was under the condition of a 12-hour light-dark cycle. Mice were housed with free access to food and water before experimentation. Mice were anesthetized with xylazine $(10 \mathrm{mg} / \mathrm{kg})$ and ketamine (75 $\mathrm{mg} / \mathrm{kg}$ ) and immobilized by placing the head in a stereotactic frame. Oxygen was supplied through a face mask during surgery. After a $10 \mathrm{~mm}$ long midline incision was made on the scalp, a $3.5 \mathrm{~mm}$ diameter opening was drilled in the right cranium $2.0 \mathrm{~mm}$ lateral to the sagittal 
suture between bregma and lambda. The surgical procedure was completed for the Sham group. For the experimental groups, a controlled cortical impact (CCI) device (PinPoint Precision Cortical Impactor PCI3000; Hatteras Instruments, Cary, NC, USA) was used to establish the TBI model. A $3.5 \mathrm{~mm}$ diameter rounded steel impactor tip was placed on the exposed intact dura. And then the cortical surface was hit vertically at an impact velocity of $1.5 \mathrm{~m} / \mathrm{s}$ to deform a depth of $1.5 \mathrm{~mm}$, and dwell time of $100 \mathrm{~ms}$. A sterile cotton gauze and pressure were used to control the bleeding of the injured cortical surface. The incision was sutured with interrupted 6.0 silk sutures after the cranial defect was sealed with sterile bone wax. The animal was placed on a heating pad for recovery from anesthesia until they regained full consciousness and then moved to their home cages.

\section{Treatments for Mice}

After TBI, mice were randomly divided into four groups $(\mathrm{n}=10)$ : Sham group, TBI+ normal saline (NS group), TBI +TNA (TNA group), and TBI+TNA+ICI group (TNA+ICI group). We injected NS as control. TNA was purchased from Sigma-Aldrich (T4952, St. Louis, MO, USA). In TNA and TNA+ICI group, TNA was administered 1 $\mathrm{h}$ after operation $(50 \mathrm{mg} / \mathrm{kg})$ by intraperitoneal injection. From day 1 to 6 post-TBI, TNA was administrated $(20 \mathrm{mg} /$ $\mathrm{kg}$ ) once a day at the same time. Mice in TNA+ICI group were treated with ICI 182,780 (20 mg/kg/d; I4409, St. Louis, MO, USA) by s.c. injection $1 \mathrm{~h}$ after TBI and once a day from day 1 to 6 post-TBI. Agar chow instead of solid chow was used since the mice were hemiplegia and hemidysesthesia after TBI.

\section{Modified Neurological Severity Score (mNSS)}

On day $0,1,3,5$, and 7 after TBI modified neurological severity score (mNSS) assessment was performed by a researcher who was blinded to the study. The NSS consists of motor (muscle status, abnormal movement), sensory (visual, tactile, proprioceptive), reflex, and balance tests as described previously. Neurological function was graded on a scale of 0 to 18 (normal score, 0; maximal deficit score, 18). A higher mNSS score indicates a more severe injury.

\section{Histological Analysis}

On day 7 after TBI, four mice from each group were sacrificed under deep anesthesia and transcardially perfused by $1 \mathrm{X}$ PBS followed by $4 \%$ paraformaldehyde, for histological analysis. The injured regions were cryoprotected with $30 \%$ sucrose for $24 \mathrm{~h}$ after $24 \mathrm{~h}$ fixation in $4 \%$ paraformaldehyde. To evaluate Ibal expression, serial $10 \mu \mathrm{m}$-thick coronal sections were processed for staining with Rabbit monoclonal to Ibal antibody (1:1000, Abcam, ab178846). Nissl staining was done for calculating neurons. Briefly frozen sections were mounted on gelatin coated slides. Sections were dried in air on slide overnight. Slides were placed into 1:1 alcohol/chloroform overnight and then rehydrated through $100 \%$ and $95 \%$ alcohol to distilled water. Slides were stained in $0.1 \%$ cresyl violet solution for 5-10 minutes. Then slides were rinsed quickly in distilled water. After that slides were differentiated in 95\% ethyl alcohol for 5-10 minutes and checked under microscope for best result. Slide were mounted with mounting medium after dehydrated in $100 \%$ alcohol and cleared in xylene. For statistical analysis every fifth slide from 50 consecutive slices i.e., 10 slices from each mouse were selected.

\section{Cell Culture and Treatment}

BV-2 cells (EOC-20 CRL-2469; ATCC), immortalized microglia cell line derived from the brain of an apparently normal 10 day old mouse, were cultured in DMEM/F12 medium with $10 \% \mathrm{FBS}$ and $1 \%$ penicillin/streptomycin at $37^{\circ} \mathrm{C}$ in a humidified atmosphere containing $95 \%$ air and $5 \%$ CO2. Cells were plated into 24 -well plates at the density of $5 \times 10^{5} /$ well. Cells were divided into four groups: control group (Ctr group), lipopolysaccharide (LPS, L4391, Sigma-Aldrich, St. Louis, MO, United States) group (LPS group), LPS+TNA group (LPS+TNA group), and LPS+TNA+ICI group (LPS+TNA+ICI group). Cells were treated in absence or presence of LPS $(1 \mu \mathrm{g} /$ $\mathrm{mL}$ ) for overnight. For LPS+TNA group and LPS+TNA + ICI group, cells were treated with concentrations of TNA at $10 \mu \mathrm{M}$ for $24 \mathrm{~h}$. Cells in LPS+TNA+ICI group were treated with ICI 182, 780 (Sigma-Aldrich, St. Louis, MO, United States) at $5 \mu \mathrm{mol} / 1$ for $24 \mathrm{~h}$. At last cells were collected for quantitative RT-PCR and Western blotting.

IL-10 knockdown was conducted by 300 nM IL-10 siRNA plasmids (sc-39,635, Santa Cruz Biotechnology, Inc.) or a nonsense scramble siRNA (sc-37,007, Santa Cruz Biotechnology, Inc.) transfection in BV-2 cells. After transfection for $24 \mathrm{~h}$, cells were treated with LPS+TNA for another $24 \mathrm{~h}$. Then cells were collected for analyzing. 


\section{RT-PCR}

On day 7 mice from each group were sacrificed for collecting fresh brain samples. Brains were dissected out and kept in nitrogen. RT-PCR was performed $\mathrm{s}$ describe before. ${ }^{32}$ Following manufacturer's instructions total RNA from lung or BV-2 cells was extracted by using RNeasy kits (Invitrogen, St. Louis, MO, USA). An on-column addition of DNase (Qiagen, Crawley, UK) was used to prevent DNA contamination according to manufacturer's instructions. RNA samples were subjected to reverse transcription using a PrimeScript RT Reagent Kit (Takara, Dalian, China). For each sample the reactions were run in triplicate in three independent experiments. Then the Rotorgene software accompanying the PCR machine was used to collect and analyze data. The sample CT values were normalized to the corresponding GAPDH CT values. $\Delta \Delta \mathrm{Ct}$ method is used to calculate the relative expression levels of each gene. The primers used were as follows: iNOS forward, 5'-CG AAACGCTTCACTTCCAA- $3^{\prime}$ and reverse, 5'-TGAGCC TATATTGCTGTGGCT-3'; TNF $\alpha$ forward, 5'-GCCTCTT CTCATTCCTGCTTG-3' and reverse, 5'-CTGATGAGAGG GAGGCCATT-3'; IL-1 $\beta$ forward, 5'-GCAACTGTTCC TGAACTCAACT-3' and reverse, 5'-ATCTTTTGGGGTCC GTCAACT-3'; CD206 forward, 5'-CTCTGTTCAGCTAT TGGACGC-3' and reverse, 5'-CGGAATTTCTGGGATT CAGCTTC-3'; Arg-1 forward, 5'-AACACGGCAGTGGC TTTAACC-3' and reverse, 5'-GGTTTTCATGTGGCGCAT TC-3'; Ym1 forward, 5'-CATGAGCAAGACTTGCGTGA
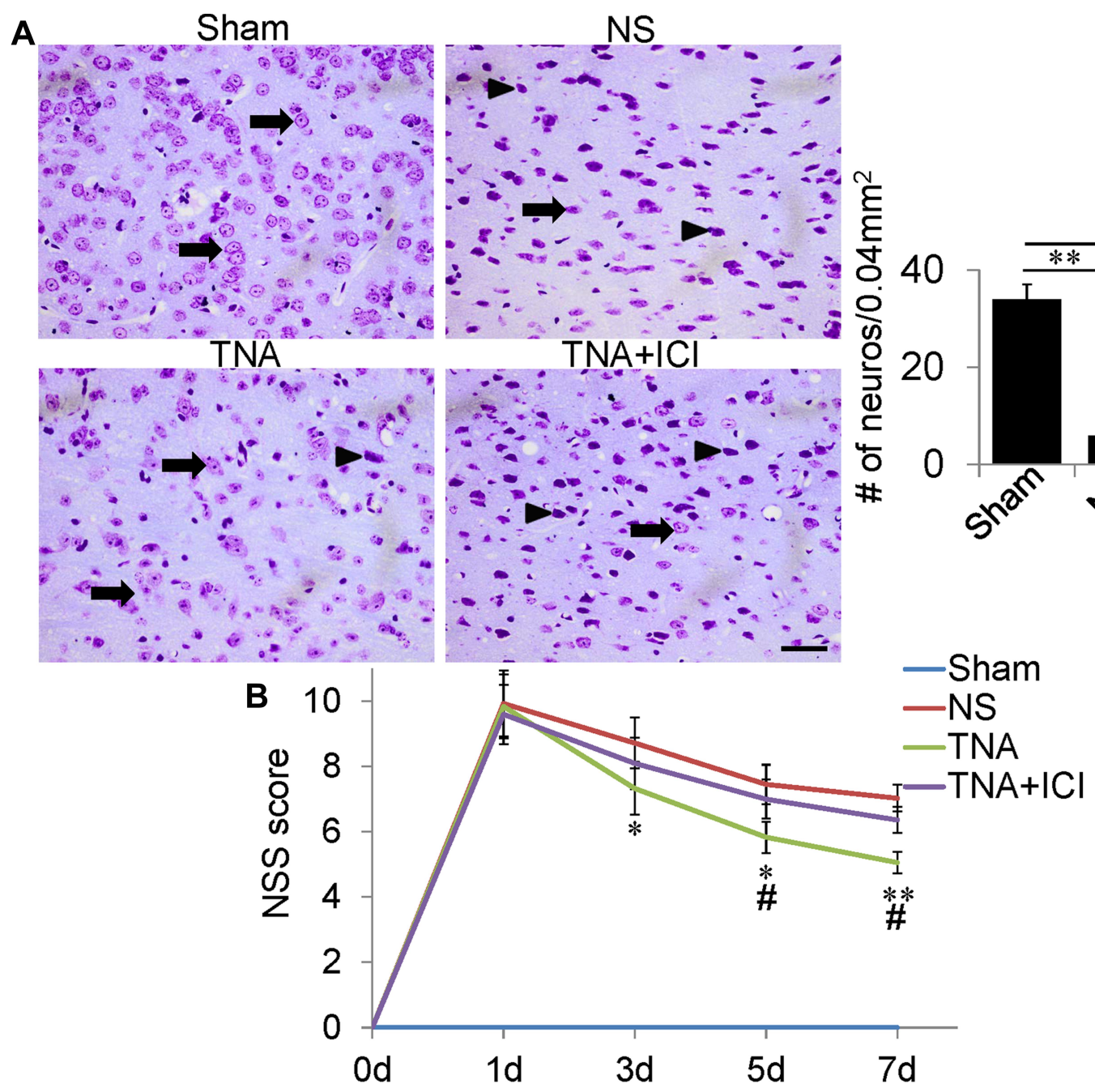

Figure I TNA treatment ameliorated neuronal loss and promoted neurological functional recovery after TBI. (A) Neurons were determined by Nissl staining. Many normal neurons (arrows) were detected in Sham group. TNA-treated mice had much more neurons on day 7 compared with NS-treated or TNA+ICl-treated mice (*P $<0.05$, $* * P<$ 0.01). (B) NSS score on day 0, I, 3, 5 and 7 after treatment. In Sham group no neurological deficit was found. Compared with NS-treated mouse, TNA-treated mice had less severe symptoms from day 3 after treatment $\left(* \mathrm{P}<0.05\right.$, $\left.{ }^{* * \mathrm{P}}<0.0 \mathrm{I}\right)$. Compared with TNA+ICl-treated mice, TNA-treated mice also showed better recovery from day 5 ( ${ }^{\#} \mathrm{P}<0.05$ ). TNA+ICl-treated mice did not recover better than NS-treated mouse within 7 days. (scale bar: $50 \mu \mathrm{m}$.).

Abbreviations: TNA, tanshinone IIA; TBI, traumatic brain injury; IC, ICI I82,780. 
C-3' and reverse, 5'-GGTCCAAACTTCCATCCT CCA-3'; IL-4 forward, 5'-TCGGCATTTTGAACGAGGTC-3' and reverse, 5'-GAAAAGCCCGAAAGAGTCTC-3'; IL-10 forward, 5'-AGAAAAGAGAGCTCCATCATGC-3' and reverse, 5'-TTATTGTCTTCCCGGCTGTACT-3'; IL-13 forward, 5'-TGAGGAGCTGAGCAACATCACACA- $3^{\prime}$ and reverse, 5'-TGCGGTTACAGAGGCCATGCAATA-3'; GAP DH forward, 5'-TCAACAGCAACTCCCACTCTTCCA-3' and reverse, 5'- ACCCTGTTGCTGTAGCCGTATTCA-3'.

\section{Western Blotting}

Brain tissues from injured regions or cells were lysed in RIPA buffer (Beijing BLKW Biotechnology Co., Ltd, Beijing, China) followed by centrifugation at $10,000 \mathrm{~g}$ for 10 minutes at $4{ }^{\circ} \mathrm{C}$. Proteins from brain tissues were electrophoresed in $10 \%$ SDS-PAGE gels under reducing conditions. After that, proteins were transferred to nitrocellulose membranes. 3\% milk in PBST was used to block unspecific bindings of antibodies. Membranes were incubated with following primary antibodies: anti-iNOS (1:1000, Santa Cruz Biotechnology, sc-7271) or anti-CD206 (1:1000, Santa Cruz Biotechnology, sc-58,986) or anti-IL-4 (1:1000, Santa Cruz Biotechnology, sc-53,084) or anti-IL-10 (1:500, Santa Cruz Biotechnology, sc-365,858) or anti-IL-13
(1:1000, Santa Cruz Biotechnology, sc-393,365). The membranes were then incubated with horseradish peroxidase (HRP)-conjugated secondary antibodies at 1:2000 for $1 \mathrm{~h}$ and were washed by PBST 3 times. An Odyssey Infrared Imaging system and software (LI-COR Bioscience) was used to evaluate the specific signals and the corresponding band intensities.

\section{Statistical Analysis}

Data are shown as mean \pm standard error of the mean (SEM). Statistical significance was analyzed by repeated measures of analysis of variance (ANOVA) followed by Bonferroni post hoc test or unpaired Student's $t$-test, if they were normally distributed (Kolmogorov-Smirnov test, $\mathrm{p}>0.05$ ). $\mathrm{P}<0.05$ was considered as statistically significant.

\section{Results}

\section{TNA Treatment Attenuated Neuronal Loss and Promoted Neurological} Functional Recovery in TBI Mice

To investigate whether TNA decreases neuronal loss in TBI mice, we treated mice from $1 \mathrm{~h}$ after TBI to day 6 with NS, TNA or combination of TNA and ICI. Mice in the Sham
A

A

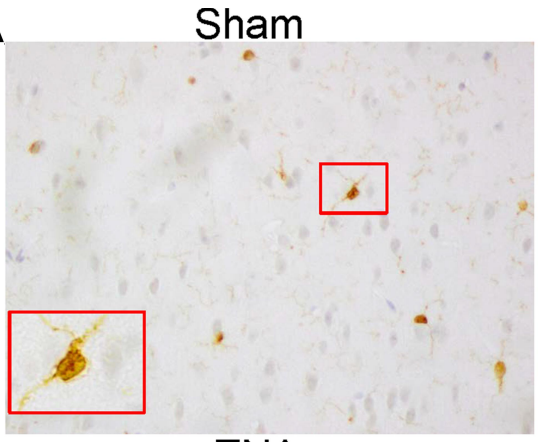

TNA

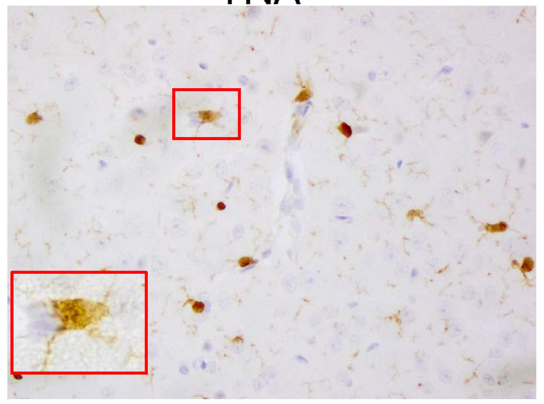

NS

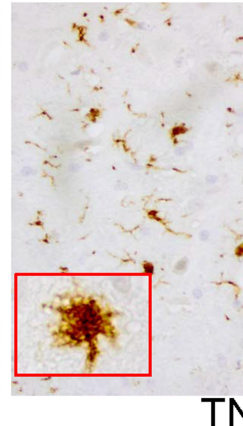

$\mathrm{TNA}+\mathrm{ICl}$

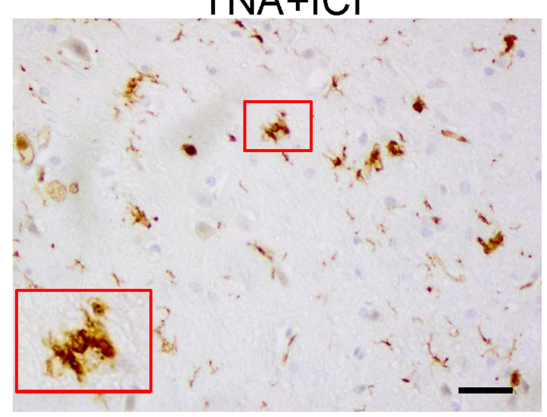

B

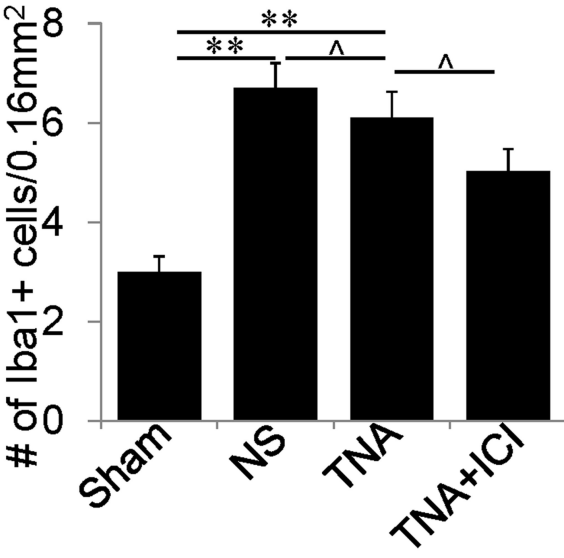

Figure 2 TNA treatment changed microglial morphology with no change in microglial numbers. (A) In Sham group some normal microglia with small cell body and long, thin branches. Compared to the microglia in Sham group there were more microglia with larger cell body and shorter, thick processes in mice treated with NS. TNA treatment obviously decreased the branches of microglia than in mice treated with NS or treated with TNA+ICl. Compared to microglia in NS-treated mice TNA+ICI treatment did not change activated microglia induced by TBI. A higher magnification picture was inserted for the red box in each picture. (B) Statistic analysis of microglial numbers (**P<0.0I, $\left.{ }^{\wedge} \mathrm{P}>0.05\right)$. (scale bar: $100 \mu \mathrm{m}$.).

Abbreviations: TNA, tanshinone IIA; TBI, traumatic brain injury; IC, ICI I82,780. 
group had many normal neurons (arrows) detected by Nissl staining (Figure 1A). However, mice in the NS group far fewer neurons with abnormal morphology (arrow heads) on day 7 after TBI (Figure 1A). TNA-treated mice had significantly more neurons on day 7 compared with that in NS-treated mice $(\mathrm{P}<0.01)$. In the TNA+ICI-treated mice, the number of neurons was significantly less than that in TNA-treated mice $(\mathrm{P}<0.05)$ (Figure 1A). To evaluate whether TNA treatment could improve sensorimotor deficit, we compared NSS scores ( $\mathrm{n}=10$ for each group). All of the mice scores were 0 before TBI (day 0 ). In the Sham group no neurological functional deficits were demonstrated in the study. There were no significant differences in NSS scores among all TBI groups on day 1 . From day 3 to day 7 neurological function was shown progressive recovery in all TBI groups. However, TNA treatment demonstrated better improvement in NSS scores comparing with NS group on day $3(\mathrm{P} 3) \quad(\mathrm{P}<0.05), \quad \mathrm{P} 5 \quad(\mathrm{P}<0.05)$ and $\mathrm{P} 7$ $(\mathrm{P}<0.01)$ (Figure 1B). ICI partially abolished the benefits of TNA treatment (Figure 1B). No significant difference on body weights was observed among the 4 groups during 7 days.

\section{TNA Inhibited Microglia Activation with the Number of Microglia Unchanged}

In Sham group, there were a few resting microglia with small cell bodies, long and thin ramified processes (Figure 2A). Microglia in NS group became activated with demonstrating enlarged bigger cell bodies and poorly ramified, short and thick processes (Figure 2A). In addition, the number of microglia was significantly increased when compared to that in Sham group (Figure 2A and B). When mice were treated with TNA, this Iba1-positive microglia had smaller cell bodies with long and thin ramified processes when

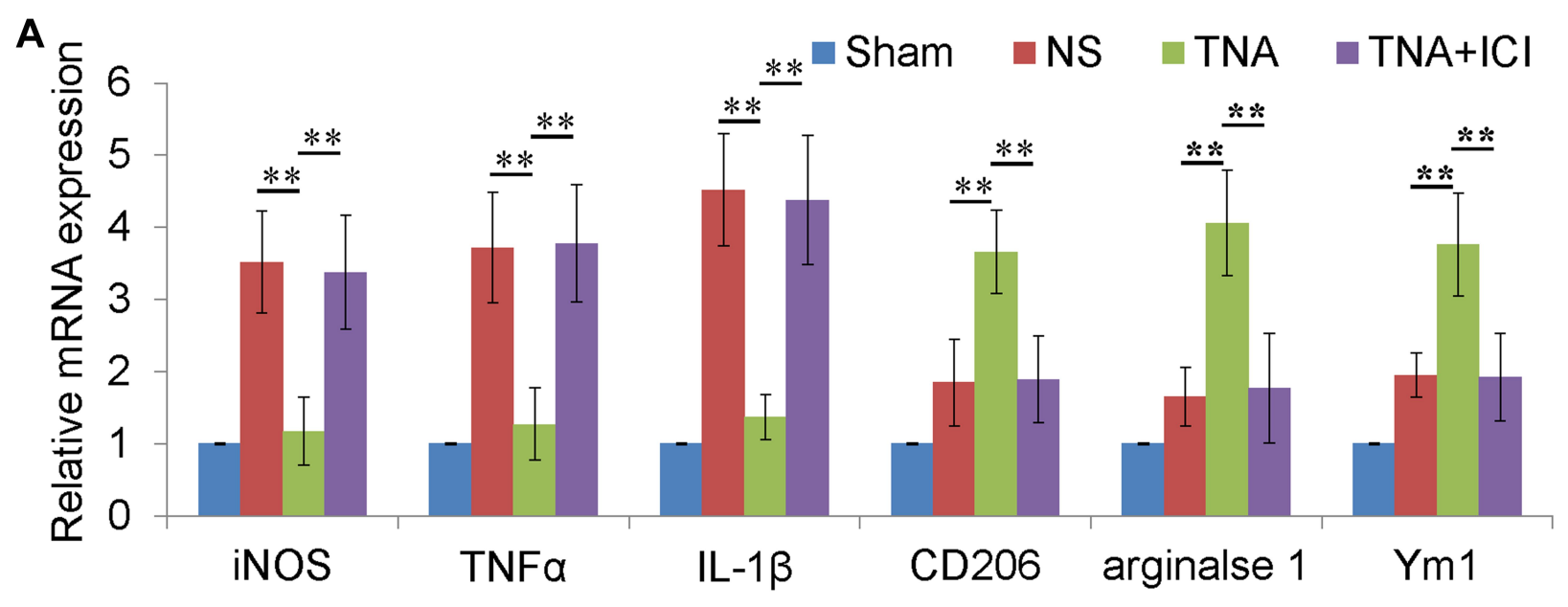

B
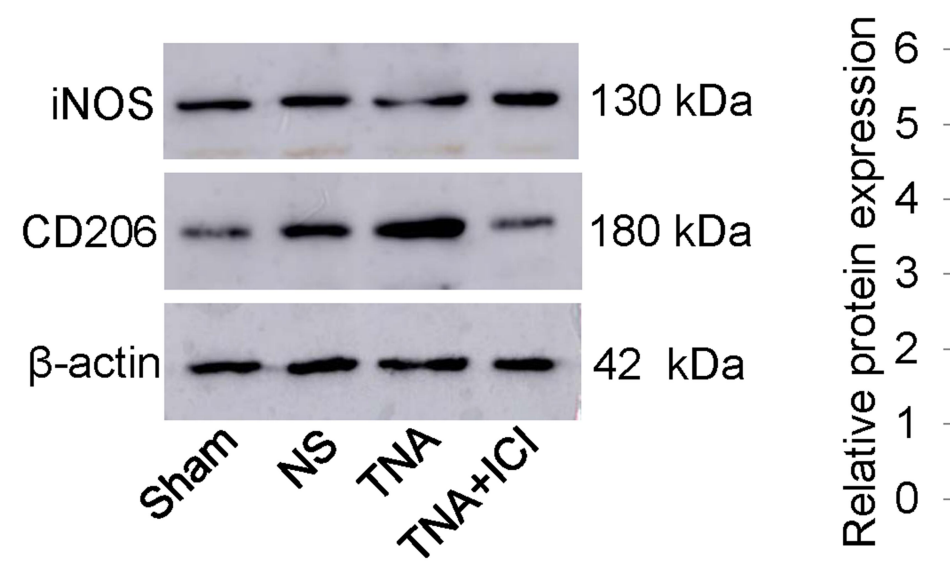

Figure 3 TNA treatment promoted microglial M2 polarization in vivo. (A) TNA treatment downregulated MI macrophage (iNOS, TNF $\alpha$ and IL-I $\beta$ ) upregulated M2 macrophage (CD206, arginase I and YmI) mRNA levels than those in NS group. No significant differences were found between TNA+ICl group and NS group. (B) Western blots for iNOS, CD206 and $\beta$-actin. TNA treatment downregulated iNOS protein about 3.2 folds and upregulated CD206 protein about 3.0 folds than those in NS group $(* * \mathrm{P}<0.01)$.

Abbreviations: TNA, tanshinone IIA; IC, ICI 182,780; iNOS, inducible nitric oxide synthase; TNF $\alpha$, tumor necrosis factor $\alpha$. 
compared to that in NS group. In TNA+ICI-treated mice, the morphology of microglia remained activated (Figure 2A). However, TNA treatment did not reduce the number of microglia when compared to that in NS-treated mice or in TNA+ICI-treated mice (Figure 2B).

\section{TNA Promoted Microglial M2 Polarization in vivo}

Microglia can be divided into two different subtypes, M1 and M2 microglia by expressing different marker proteins. TNF $\alpha$, iNOS and IL- $1 \beta$ are mainly expressed in M1 microglia. M2 microglia express CD206, Arginase 1, and Ym1. M1 and M2 marker mRNAs were both upregulated in NS group. M1 marker mRNAs were increased higher than M2 marker mRNAs (Figure 3A). TNA treatment significantly downregulated M1 macrophage maker
mRNA level and upregulated M2 macrophage marker mRNA level than those in NS or in TNA+ICI group $(\mathrm{P}<0.01)$ (Figure 3A). To confirm the change in protein level, Western blotting for iNOS and CD206 was performed. The results demonstrated that TNA treatment downregulated iNOS protein about 3.2 folds and upregulated CD206 protein about 3.0 folds than those in NS group $(\mathrm{P}<0.01)$ (Figure $3 \mathrm{~B})$.

\section{TNA Treatment Switched Microglial MI to M2 Polarization in vitro}

To further investigate whether TNA treatment switch microglial M1 to M2 polarization, BV-2 cells were cultured and activated by LPS. Notably, strong M1 microglial marker mRNAs were induced by LPS (Figure 4A). After TNA treatment M1 marker mRNAs were decreased, and

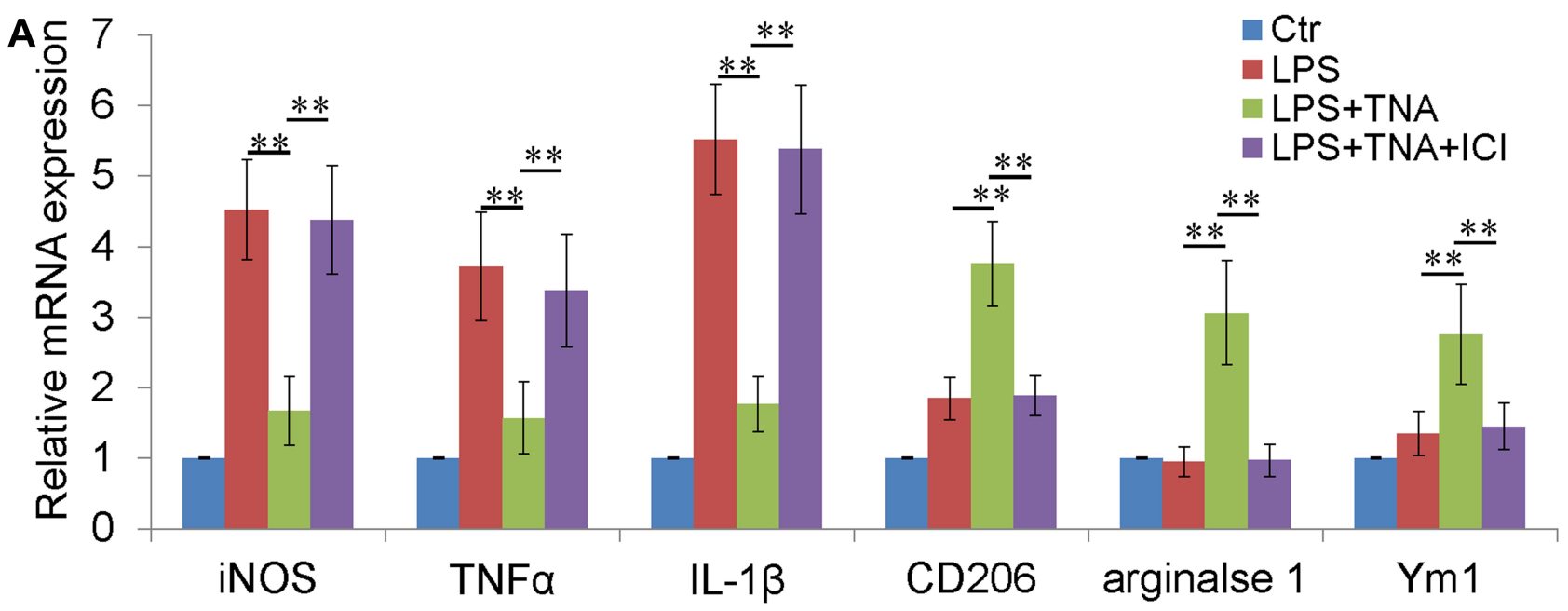

\section{B}
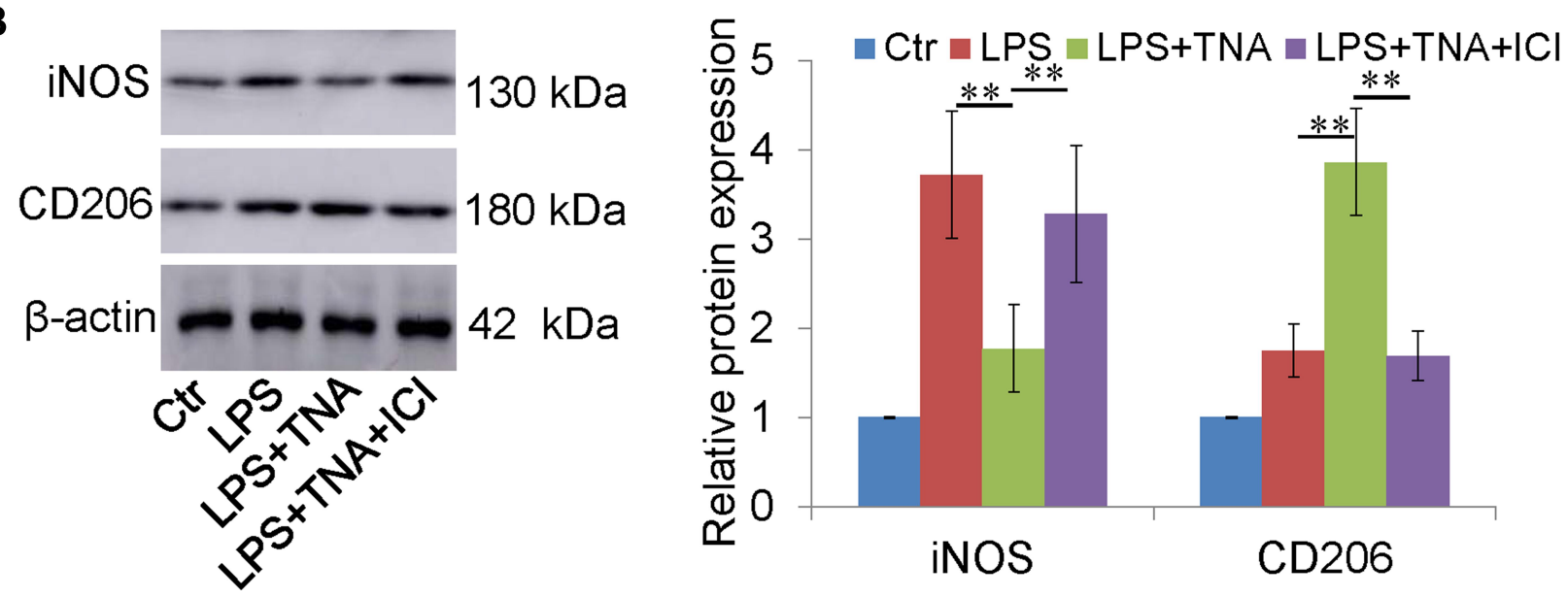

Figure 4 TNA treatment inhibited MI microglia and promoted M2 microglia in vitro. (A) TNA treatment decreased MI macrophage (iNOS, TNF $\alpha$ and IL-I $\beta$ ) increased M2 macrophage (CD206, arginase I and YmI) mRNA levels than those in LPS group. No significant changes were found between TNA+ICl group and LPS group. (B) Western blots for iNOS, CD206 and $\beta$-actin. TNA treatment decreased iNOS protein about 2.1 folds and upregulated CD206 protein about 2.2 folds than those in LPS group $(* * \mathrm{P}<0.0 \mathrm{I})$.

Abbreviations: TNA, tanshinone IIA; IC, ICI 182,780; iNOS, inducible nitric oxide synthase; TNF $\alpha$, tumor necrosis factor $\alpha$. 
M2 marker mRNAs were increased. As expected, the effect of TNA on microglial M2 polarization was blocked by ICI (Figure 4A). Western blotting for iNOS and CD206 was performed to confirm the express on protein level. The results showed that TNA treatment decreased iNOS protein about 2.1 folds and upregulated CD206 protein about 2.2 folds than those in LPS group $(\mathrm{P}<0.01)$ (Figure $4 \mathrm{~B})$.

TNA Treatment Upregulated IL- 10 Expression and IL-I0 Knockdown Abolished Microglial M2 Polarization by TNA in vitro

IL-4, IL-10 and IL-13 were reported to promote microglial M2 polarization. ${ }^{33}$ To determine whether TNA could regulate IL-4, IL-10 and IL-13, the mRNA and protein expressions of IL-4, IL-10 and IL-13 were measured. TNA treatment increased IL-10 mRNA about 2.5 folds than that in LPS group $(\mathrm{P}<0.01)$ (Figure 5A). There were no significant changes of IL-4 and IL-13 mRNA (Figure 5B). On protein level IL-10 were upregulated about 2.3 folds by TNA treatment when compared to LPS treatment $(\mathrm{P}<0.01)$. No significantly changes of IL-4 and IL-13 on protein level (Figure 5B). Further studies were then conducted to evaluate the role of IL-10 upregulated by TNA on microglia M1/M2 polarization. First Western blot for IL-10 confirmed that IL-10 siRNA efficiently knocked down IL-10 expression around $85 \%(\mathrm{P}<0.01)$ (Figure 6A). Then mRNA for M1 microglial markers and M2 microglial markers demonstrated that the effect of TNA on TNA on microglial M2 polarization was reversed by IL-10 knockdown (Figure 6B).

A
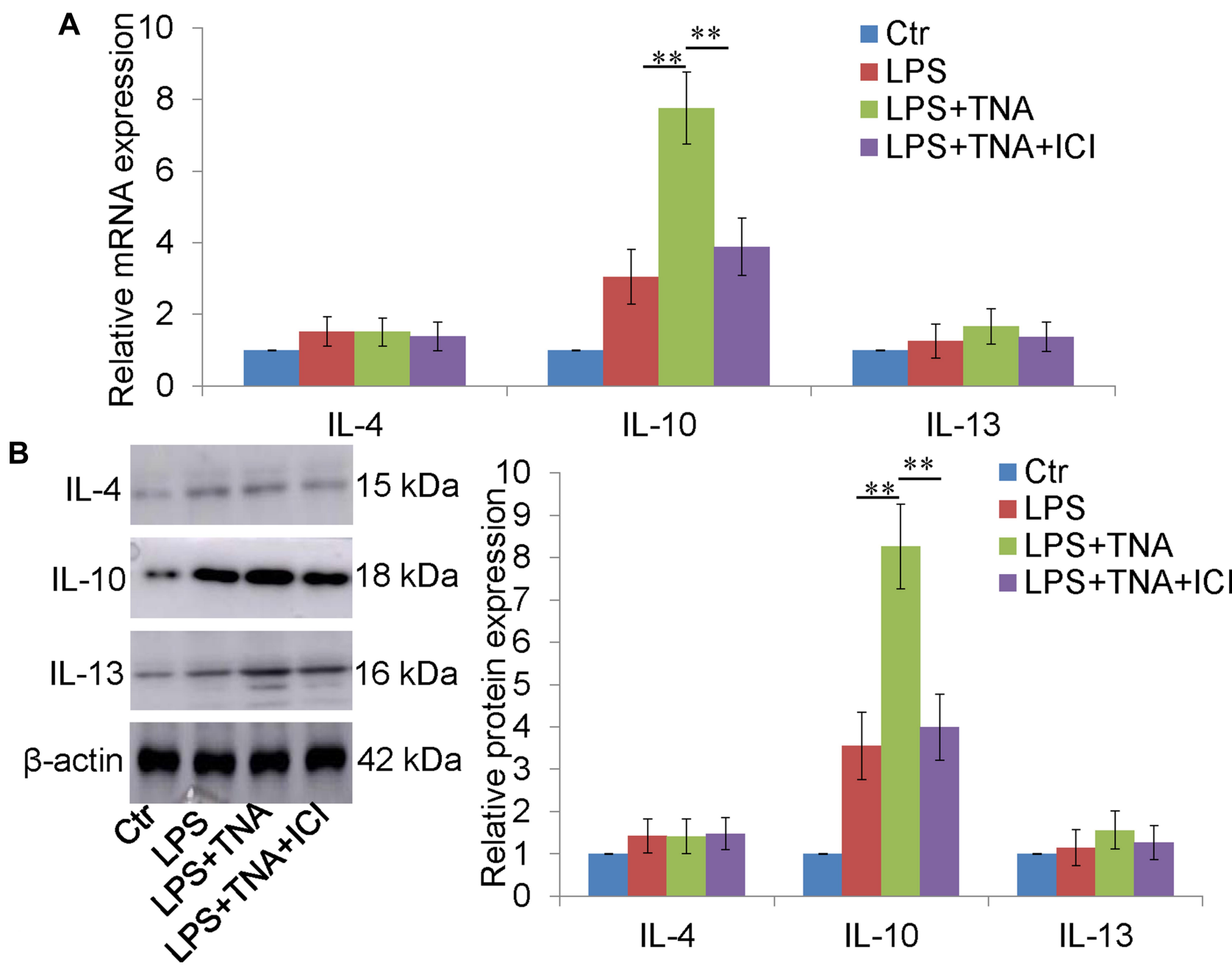

Figure 5 IL- 10 knockdown abolished the effect of TNA on microglial M2 polarization in vitro. (A) Western blot for IL- 10 and $\beta$-actin. It showed that IL- 10 siRNA knocked down around $85 \%$ of IL- 10 expression induced by LPS. (B) qPCR for MI microglia (iNOS, TNF $\alpha$ and IL-I $\beta$ ) M2 microglia (CD206, arginase I and YmI) demonstrated that the effect of TNA on TNA on microglial M2 polarization was reversed by IL-10 knockdown $(* * P<0.01$, ' $\mathrm{P}>0.05)$.

Abbreviations: TNA, tanshinone IIA; LPS, lipopolysaccharide; iNOS, inducible nitric oxide synthase; TNF $\alpha$, tumor necrosis factor $\alpha$. 


\section{Discussion}

In the present study, we investigated whether TNA shifts the M1 pro-inflammatory microglia phenotype to the M2 anti-inflammatory microglia state, thus protecting neurons and promoting functional recovery in a mouse model of TBI. The results demonstrated that TNA treatment led to a better neurological functional recovery by increasing M2 microglia. The findings of this study further proved that the ER $\beta$ antagonist abolished TNA-promoted M2 polarization. At last, we proved that TNA treatment upregulated IL-10 expression which contributed to the effect of TNA on microglial M2 polarization. Together, these studies demonstrated that TNA promoted microglial M2 polarization via
ER $\beta /$ IL-10 pathway. The results presented here provide new insights into the role of TNA for treating TBI.

In CNS microglia and astrocytes are two types of resident glial cells that communicating each other under homeostatic conditions and under affecting by pathology. ${ }^{32}$ It has been reported that microglia in CNS do not express ER $\alpha{ }^{28}$ The effect of TNA on microglial M2 polarization abolished by ICI, an estrogen receptors antagonist, is via blocking ER $\beta$ instead of via $\mathrm{ER} \alpha$.

TBI causes high rates of disability and mortality and is responsible for a major public health burden. ${ }^{34}$ In order to explore the underlying pathogenesis and evaluate potential therapies several TBI models, including CCI, weight-drop

A

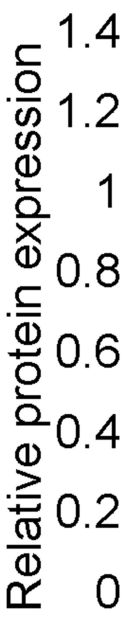

B
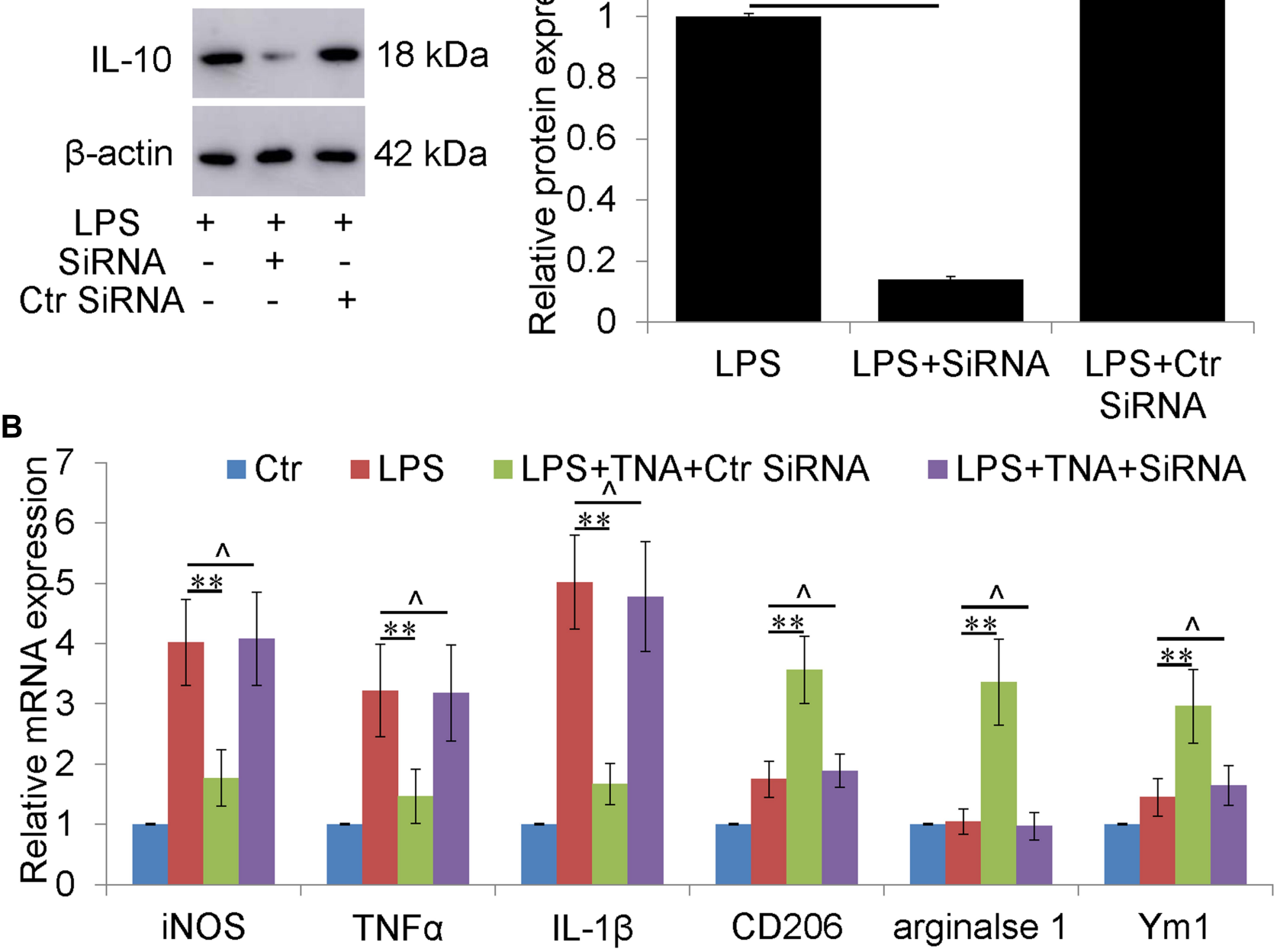

Figure 6 TNA treatment upregulated IL-10, not IL-4 and IL-I3 in vitro. (A) TNA treatment significantly increased IL-10 mRNA level than that in LPS group. No significant changes of IL-4 and IL- I 3 were found. (B) Western blots for IL-4, IL-I0, IL-I 3 and $\beta$-actin. TNA treatment upregulated IL-I0 protein about 2.3 folds than that in LPS group. IL-4 and IL-I 3 were not significantly changed $(* * \mathrm{P}<0.0 \mathrm{I})$.

Abbreviation: TNA, tanshinone IIA. 
injury, fluid percussion injury, blast brain injury, and penetrating brain injury, have been used in mice. ${ }^{35-38}$ In our study, CCI brain injury model was used due to its ease of operation and high accuracy compared with that of other models. We confirmed our CCI model was success with mNSS, loss of neurons and activated microglia. In this study Nissl staining, a relatively simple technique for staining the neurons, was used to identify neurons. The number of stained neurons was significantly less in the NS-treated mice than that in intact mice (Sham group).

Microglia, the resident macrophages in the brain, respond to external injury by shifting phenotypes and related functions. M1 microglia represent the classical activation with expression of iNOS, IL- $1 \beta$ and TNF $\alpha$ which contribute to the surrounding neuronal damage. M2 microglia represent alternative activation with expression of arginase 1, CD206, Ym1, Fizz1. ${ }^{39}$ Following TBI M1 microglia is rapidly induced and maintained at the site of the injury, whereas M2 microglia is transiently expressed after injury. ${ }^{40,41}$ Based on the functional plasticity of microglia, the treatment of CNS injury through promoting a shift from the M1 to M2 phenotype has been shown promising. ${ }^{41}$ The present study indicated that TNA inhibited M1 microglia activation-mediated neuroinflammation by downregulating iNOS, IL-1 $\beta$ and $\mathrm{TNF} \alpha$ and promoted M2 microglia by upregulating arginase $1, \mathrm{CD} 206, \mathrm{Ym} 1$. These results were partially consistent with the previous studies that TNA attenuated traumatic injury of the spinal cord by decreasing the production of pro-inflammatory cytokines (TNF- $\alpha$, IL-1 $\beta$, and IL-6) and iNOS in rats. ${ }^{26}$

IL-4, IL-10 and IL-13 are able to induce microglial M2 polarization. $^{42} \mathrm{BV}-2$ cells were introduced to investigate which interleukin(s) is/are contributing to the effect of TNAinduced microglial M2 polarization. Further analysis of cascade signaling events underlying TNA-mediated microglial M2 polarization demonstrated that the upregulated IL-10 by TNA was responsible for effect of TNA on microglial M2 polarization. IL-10 knockdown abolished TNA-induced microglial M2 polarization further confirmed the result. In this study IL-4 and IL-13 were not found to contribute to the microglial M2 polarization. These data suggested that TNAmediated microglial M2 polarization was dependent on the activation of ER $\beta / \mathrm{IL}-10$ signaling.

In summary, this study demonstrated that TNA administration switched the microglial polarization toward M2 state via activating ER $\beta / \mathrm{IL}-10$ signaling activation, which was accompanied by the reduced neuronal loss and decreased neuroinflammatory response. The current results supported the potential pharmaceutical application of TNA in TBI and other neuroinflammatory-related neurological disorders.

\section{Conclusion}

This study illustrates that TNA promoted microglial polarization toward the M2 phenotype through activating ER $\beta$ / IL-10 signaling. These findings provide new evidence that TNA might be considered as a potent agent for the treatment of TBI and neuroinflammatory disorders.

\section{Acknowledgments}

All authors have read and agreed the submission and publication. All experiments were approved by the Ethics Committee of Guizhou Provincial People's Hospital, Guizhou, China.

\section{Author Contributions}

All authors made a significant contribution to the work reported, whether that is in the conception, study design, execution, acquisition of data, analysis and interpretation, or in all these areas; took part in drafting, revising or critically reviewing the article; gave final approval of the version to be published; have agreed on the journal to which the article has been submitted; and agree to be accountable for all aspects of the work.

\section{Disclosure}

The authors declare no conflicts of interest.

\section{References}

1. Yang WH, Chen PC, Wang TC, Kuo TY, Cheng CY, Yang YH. Endocrine dysfunction following traumatic brain injury: a 5-year follow-up nationwide-based study. Sci Rep. 2016;6(1):32987. doi:10.1038/srep32987

2. Dewan MC, Rattani A, Gupta S, et al. Estimating the global incidence of traumatic brain injury. $J$ Neurosurg. 2018;1:1-18.

3. Jiang JY, Gao GY, Feng JF, et al. Traumatic brain injury in China. Lancet Neurol. 2019;18(3):286-295. doi:10.1016/S1474-4422(18) 30469-1

4. Rubiano A, Carney N, Chesnut R, Puyana J. Global neurotrauma research challenges and opportunities. Nature. 2015;527(7578):S193S197. doi:10.1038/nature16035

5. Raghupathi R. Cell death mechanisms following traumatic brain injury. Brain Pathol. 2004;14(2):215-222. doi:10.1111/j.1750-3639. 2004.tb00056.x

6. Narayan RK, Michel ME, Ansell B, et al. Clinical trials in head injury. $J$ Neurotrauma. 2002;19(5):503-557. doi:10.1089/08977150 2753754037

7. Graham NS, Sharp DJ. Understanding neurodegeneration after traumatic brain injury: from mechanisms to clinical trials in dementia. J Neurol Neurosurg Psychiatry. 2019;90(11):1221-1233. doi:10.1136/ jnnp-2017-317557 
8. Dinet V, Petry KG, Badaut J. Brain-immune interactions and neuroinflammation after traumatic brain injury. Front Neurosci. 2019;13:1178. doi:10.3389/fnins.2019.01178

9. Rhodes J. Peripheral immune cells in the pathology of traumatic brain injury? Curr Opin Crit Care. 2011;17(2):122-130. doi:10.1097/MCC.0b013e3283447948

10. Skaper SD, Facci L, Giusti P. Neuroinflammation, microglia and mast cells in the pathophysiology of neurocognitive disorders: a review. CNS Neurol Disord Drug Targets. 2015;13(10):1654-1666. doi:10.2174/1871527313666141130224206

11. Sica A, Mantovani A. Macrophage plasticity and polarization: in vivo veritas. J Clin Invest. 2012;122(3):787-795. doi:10.1172/JCI59643

12. Colton CA. Heterogeneity of microglial activation in the innate immune response in the brain. $J$ Neuroimmune Pharmacol. 2009;4:399-418.

13. Qin L, Liu Y, Wang $T$, et al. NADPH oxidase mediates lipopolysaccharide-induced neurotoxicity and proinflammatory gene expression in activated microglia. J Biol Chem. 2004;279 (2):1415-1421. doi:10.1074/jbc.M307657200

14. Liu R, Liao XY, Tang JC, et al. BpV(pic) confers neuroprotection by inhibiting M1 microglial polarization and MCP-1 expression in rat traumatic brain injury. Mol Immunol. 2019;112:30-39. doi:10.1016/j. molimm.2019.04.010

15. Chio CC, Lin MT, Chang CP. Microglial activation as a compelling target for treating acute traumatic brain injury. Curr Med Chem. 2015;22(6):759-770. doi:10.2174/0929867321666141106124657

16. Loane DJ, Stoica BA, Tchantchou F, et al. Novel mGluR5 positive allosteric modulator improves functional recovery, attenuates neurodegeneration, and alters microglial polarization after experimental traumatic brain injury. Neurotherapeutics. 2014;11(4):857-869. doi:10.1007/s13311-014-0298-6

17. Yao X, Liu S, Ding W, et al. TLR4 signal ablation attenuated neurological deficits by regulating microglial M1/M2 phenotype after traumatic brain injury in mice. $J$ Neuroimmunol. 2017;310:38-45. doi:10.1016/j.jneuroim.2017.06.006

18. Morganti JM, Jopson TD, Liu S, et al. CCR2 antagonism alters brain macrophage polarization and ameliorates cognitive dysfunction induced by traumatic brain injury. J Neurosci. 2015;35(2):748-760. doi:10.1523/JNEUROSCI.2405-14.2015

19. Zanier ER, Pischiutta F, Riganti L, et al. Bone marrow mesenchymal stromal cells drive protective M2 microglia polarization after brain trauma. Neurotherapeutics. 2014;11(3):679-695. doi:10.1007/ s13311-014-0277-y

20. Gao S, Mao F, Zhang B, et al. Mouse bone marrow-derived mesenchymal stem cells induce macrophage M2 polarization through the nuclear factor- $\kappa \mathrm{B}$ and signal transducer and activator of transcription 3 pathways. Exp Biol Med (Maywood). 2014;239(3):366-375. doi:10.1177/1535370213518169

21. Chen Z, Xu H. Anti-inflammatory and immunomodulatory mechanism of tanshinone IIA for atherosclerosis. Evid Based Complement Alternat Med. 2014;2014:267976. doi:10.1155/2014/267976

22. Gao H, Huang L, Ding F, et al. Simultaneous purification of dihydrotanshinone, tanshinone I, cryptotanshinone, and tanshinone IIA from Salvia miltiorrhiza and their anti-inflammatory activities investigation. Sci Rep. 2018;8(1):8460. doi:10.1038/s41598-01826828-0

23. Shu M, Hu XR, Hung ZA, Huang DD, Zhang S. Effects of tanshinone IIA on fibrosis in a rat model of cirrhosis through heme oxygenase-1, inflammation, oxidative stress and apoptosis. Mol Med Rep. 2016;13(4):3036-3042. doi:10.3892/mmr.2016.4886

24. Chen W, Li X, Guo S, et al. Tanshinone IIA harmonizes the crosstalk of autophagy and polarization in macrophages via miR-375/KLF4 pathway to attenuate atherosclerosis. Int Immunopharmacol. 2019;70:486-497. doi:10.1016/j.intimp.2019.02.054
25. Cheung DW, Koon CM, Wat E, et al. A herbal formula containing roots of Salvia miltiorrhiza (Danshen) and Pueraria lobata (Gegen) inhibits inflammatory mediators in LPS-stimulated RAW 264.7 macrophages through inhibition of nuclear factor $\kappa \mathrm{B}(\mathrm{NF} \kappa \mathrm{B})$ pathway. $J$ Ethnopharmacol. 2013;145(3):776-783. doi:10.1016/j. jep.2012.12.011

26. Yin X, Yin Y, Cao FL, et al. Tanshinone IIA attenuates the inflammatory response and apoptosis after traumatic injury of the spinal cord in adult rats. PLoS One. 2012;7:e3838.

27. Gong G, Gu Y, Zhang Y, Liu W, Li L, Li J. Tanshinone IIA alleviates oxidative damage after spinal cord injury in vitro and in vivo through up-regulating miR-124. Life Sci. 2019;216:147-155. doi:10.1016/j. lfs.2018.11.046

28. Wu WF, Tan XJ, Dai YB, Krishnan V, Warner M, Gustafsson JÅ. Targeting estrogen receptor $\beta$ in microglia and T cells to treat experimental autoimmune encephalomyelitis. Proc Natl Acad Sci US A. 2013;110(9):3543-3548. doi:10.1073/pnas.1300313110

29. Saijo K, Collier JG, Li AC, Katzenellenbogen JA, Glass CK. An ADIOL-ER $\beta$-CtBP transrepression pathway negatively regulates microglia-mediated inflammation. Cell. 2011;145(4):584-595. doi:10.1016/j.cell.2011.03.050

30. Wang L, Zhao RP, Song XY, Wu WF. Targeting ER $\beta$ in macrophage reduces crown-like structures in adipose tissue by inhibiting osteopontin and HIF-1 $\alpha$. Sci Rep. 2019;9(1):15762. doi:10.1038/s41598019-52265-8

31. Deng X, Xing X, Sun G, et al. Guanxin Danshen formulation protects against myocardial ischemia reperfusion injury-induced left ventricular remodeling by upregulating estrogen receptor $\beta$. Front Pharmacol. 2017;8:777. doi:10.3389/fphar.2017.00777

32. Jin ML, Zou ZH, Tao $\mathrm{T}$, et al. Effect of the recombinant adenovirus-mediated HIF-1 alpha on the expression of VEGF in the hypoxic brain microvascular endothelial cells of rats. Neuropsychiatr Dis Treat. 2020;16:397-406. doi:10.2147/NDT.S238616

33. Oishi S, Takano R, Tamura S, et al. M2 polarization of murine peritoneal macrophages induces regulatory cytokine production and suppresses T-cell proliferation. Immunology. 2016;149(3):320-328. doi:10.1111/imm.12647

34. Fang J, Zhu Y, Wang $\mathrm{H}$, et al. Baicalin protects mice brain from apoptosis in traumatic brain injury model through activation of autophagy. Front Neurosci. 2019;12:1006. doi:10.3389/ fnins.2018.01006

35. Jing Y, Yang D, Fu Y, et al. Neuroprotective effects of serpina3k in traumatic brain injury. Front Neurol. 2019;10:1215. doi:10.3389/ fneur.2019.01215

36. Wang ZF, Gao C, Chen W, et al. Salubrinal offers neuroprotection through suppressing endoplasmic reticulum stress, autophagy and apoptosis in a mouse traumatic brain injury model. Neurobiol Learn Mem. 2019;161:12-25. doi:10.1016/j.nlm.2019.03.002

37. Bhowmick S, D’Mello V, Caruso D, Wallerstein A, Abdul-Muneer PM. Impairment of pericyte-endothelium crosstalk leads to blood-brain barrier dysfunction following traumatic brain injury. Exp Neurol. 2019;317:260-270. doi:10.1016/j.expneurol.2019. 03.014

38. Sajja VSSS, Jablonska A, Haughey N, et al. Sphingolipids and microRNA changes in blood following blast traumatic brain injury: an exploratory study. $J$ Neurotrauma. 2018;35(2):353-361. doi:10.1089/neu.2017.5009

39. Varnum MM, Ikezu T. The classification of microglial activation phenotypes on neurodegeneration and regeneration in Alzheimer's disease brain. Arch Immunol Ther Exp (Warsz). 2012;60:251-266.

40. Kumar A, Alvarez-Croda DM, Stoica BA, Faden AI, Loane DJ. Microglial/macrophage polarization dynamics following traumatic brain injury. J Neurotrauma. 2016;33(19):1732-1750. doi:10.1089/ neu. 2015.4268 
41. Loane DJ, Kumar A. Microglia in the TBI brain: the good, the bad, and the dysregulated. Exp Neurol. 2016;275(Pt 3):316-327. doi:10.1016/j.expneurol.2015.08.018
42. Liu YC, Zou XB, Chai YF, Yao YM. Macrophage polarization in inflammatory diseases. Int $J$ Biol Sci. 2014;10(5):520-529. doi:10.7150/ijbs. 8879

\section{Publish your work in this journal}

Neuropsychiatric Disease and Treatment is an international, peerreviewed journal of clinical therapeutics and pharmacology focusing on concise rapid reporting of clinical or pre-clinical studies on a range of neuropsychiatric and neurological disorders. This journal is indexed on PubMed Central, the 'PsycINFO' database and CAS, and is the official journal of The International Neuropsychiatric Association (INA). The manuscript management system is completely online and includes a very quick and fair peer-review system, which is all easy to use. Visit http://www.dovepress.com/testimonials.php to read real quotes from published authors. 